\title{
Industrial applications of extended output-only Blind Source Separation techniques
}

\author{
C. Rutten, V.H. Nguyen, J.C. Golinval \\ University of Liège, Aerospace \& Mechanical Engineering Department, Structural Dynamic \\ Research Group \\ E-mail of the corresponding author: christophe.rutten@ulg.ac.be
}

\begin{abstract}
In the field of structural health monitoring or machine condition monitoring, most vibration based methods reported in the literature require to measure responses at several locations on the structure. In machine condition monitoring, the number of available vibration sensors is often small and it is not unusual that only one single sensor is used to monitor a machine. This paper presents industrial applications of two possible extensions of output-only Blind Source Separation (BSS) techniques, namely Principal Component Analysis (PCA) and Second Order Blind Identification (SOBI). Through the use of block Hankel matrices, these methods may be used when a reduced set of sensors or even one single sensor is available. The objective is to address the problem of fault detection in mechanical systems using subspace-based methods. The detection is achieved by comparing the subspace features between the reference and a current state using the concept of angular coherence between subspaces.
\end{abstract}

Key words: Health monitoring, Fault detection, Subspace, PCA, SOBI, Hankel matrices.

\section{Introduction}

Blind source separation (BSS) techniques allow to recover a set of underlying sources from observations without any knowledge of the mixing process or the sources. BSS techniques were shown useful for modal identification [1], damage detection and condition monitoring [2] from output-only data. In the BSS family, on can cite for example the Principal Component Analysis (PCA) and the Second Order Blind Identification (SOBI) [3].

A drawback of many BSS techniques is the need for several sensors. However, through the use of Hankel matrices these methods can be extended to damage detection problem where only one sensor signal is available [4]. 


\section{Principal Component Analysis}

Let us assume that a dynamical system is characterized by a set of vibration features collected in the matrix $\mathbf{X} \in \mathfrak{R}^{(m \times N)}$, where $m$ is the number of sensors and $N$ is the number of samples.

PCA provides a linear mapping of data from the original dimension $m$ to a lower dimension $p$. In practice, PCA is often computed by a Singular Value Decomposition (SVD) of matrix $\mathbf{X}$, i.e.

$$
\mathbf{X}=\mathbf{U} \Sigma \mathbf{V}^{\mathrm{T}}
$$

where $\mathbf{U}$ and $\mathbf{V}$ are orthonormal matrices, the columns of $\mathbf{U}$ define the principal components (PCs). The order $p$ of the system is determined by selecting the first $p$ non-zero singular values in $\Sigma$ which have a significant magnitude ("energy") as depicted in [2].

\section{Second Order Blind Identification}

As described in [3], SOBI considers the observed signals as a noisy instantaneous linear mixture of source signals. In many situations, multidimensional observations are represented:

$$
\mathbf{X}(t)=\mathbf{Y}(t)+\boldsymbol{\sigma}(t)=\mathbf{A S}(t)+\boldsymbol{\sigma}(t)
$$

Where:

- $\mathbf{X}(t)=\left[x_{1}(t), \ldots, x_{m}(t)\right]^{\mathrm{T}}$ is an instantaneous mixture of source signals and noise.

- $\mathbf{S}(t)=\left[s_{1}(t), \ldots, s_{p}(t)\right]^{\mathrm{T}}$ contains the signal from $p$ narrow band sources $(p<m)$.

- $\mathbf{Y}(t)=\left[y_{1}(t), \ldots, y_{m}(t)\right]^{\mathrm{T}}$ contains the sources assembly at a time $t$.

A is the transfer matrix between the sources and the sensor, called the mixing matrix. Under certain conditions, the mixing matrix identifies to the modal matrix of the structure and the sources correspond to normal coordinates [1]. $\boldsymbol{\sigma}(t)$ is the noise vector, modeled as a stationary white, zero-mean random process. Furthermore it is assumed to be independent of the sources. 
The SOBI method aims at recovering the mixing matrix and the sources from their mixture. More details about this technique can be found in [3].

\section{Hankel Matrices}

Block Hankel matrices play an important role in subspace system identification [5]. Those matrices characterize the dynamics of the analyzed signals and have been used for modal identification and damage detection $[4,7,8]$.

The covariance-driven block Hankel matrix is defined as follows:

$$
\mathbf{H}_{r, c}=\left[\begin{array}{cccc}
\Delta_{1} & \Delta_{2} & \cdots & \Delta_{c} \\
\Delta_{2} & \Delta_{1} & \cdots & \Delta_{c+1} \\
\vdots & \vdots & \ddots & \vdots \\
\Delta_{r} & \Delta_{r+1} & \cdots & \Delta_{r+c+1}
\end{array}\right],(c \geq r)
$$

where $r, c$ are user-defined parameters $\left(r=c\right.$, in this paper) and $\Delta_{i}$ represents the output covariance matrix.

The data-driven Hankel matrix is defined as:

$$
\mathbf{H}_{1,2 i}=\left[\begin{array}{cccc}
x_{1} & x_{2} & \cdots & x_{j} \\
x_{2} & x_{3} & \cdots & x_{j+1} \\
\vdots & \vdots & \ddots & \vdots \\
x_{j} & x_{j+1} & \cdots & x_{i+j+1} \\
\hdashline x_{j+1} & x_{j+2} & \cdots & x_{i+j} \\
x_{j+2} & x_{j+3} & \cdots & x_{i+j+1} \\
\vdots & \vdots & \ddots & \vdots \\
x_{2 i} & x_{2 i+1} & \cdots & x_{2 i+j-1}
\end{array}\right] \equiv\left(\frac{\mathbf{X}_{p}}{\mathbf{X}_{f}}\right) \equiv \frac{\text { "past" }}{\text { "future" }}
$$

where $2 i$ is a user-defined number of row blocks, each block contains $m$ rows (number of measurement sensors), $j$ is the number of columns (practically $j=N-2 i+1, N$ is the number of sampling points).

Enhanced-PCA (EPCA) and Enhanced-SOBI (ESOBI) techniques, as proposed in [4], consider the Hankel matrices, instead of the observation matrix $\mathbf{X}$, as input matrix. It can be shown that this procedure not only allows to enhance identification and detection but also permits the application of both methods to problems where only one sensor is available. 


\section{Damage detection problem}

Damage detection is based on the concept of subspace angle introduced by Golub and Van Loan [6]. Subspace angles can be used as an indicator to quantify the spatial coherence between two data sets resulting from observation of a vibration system $[2,4]$.

The change in the system dynamics may then be detected by monitoring the angular coherence between subspaces estimated from a reference observation set and from the observation set of a current state of the system. A state is considered as reference if the system operates in normal conditions (i.e. damage does not exist).

In the case of EPCA, the considered subspaces are the active subspaces built by the first $p$ columns of $\mathbf{U}$ while for ESOBI, the subspaces are built by the first columns of the mixing matrix $\mathbf{A}$.

\section{Industrial applications}

\subsection{Quality control of electro-mechanical devices}

This industrial application concerns the case of electro-mechanical devices for which the overall quality at the end of the assembly line has to be assessed. A set of nine rotating devices was instrumented with two accelerometers: one triaxial accelerometer was located on the flank of the component, and one monoaxial on the top. Among this set of nine devices, five of them are known to be healthy (referenced OK-0 $\rightarrow$ OK-4) and the other four are faulty (NOK-1 $\rightarrow$ NOK-4). As it was shown in [8] that the detection is the best in the $\mathrm{Y}$ direction, the data in this same direction are used here to test the methods.
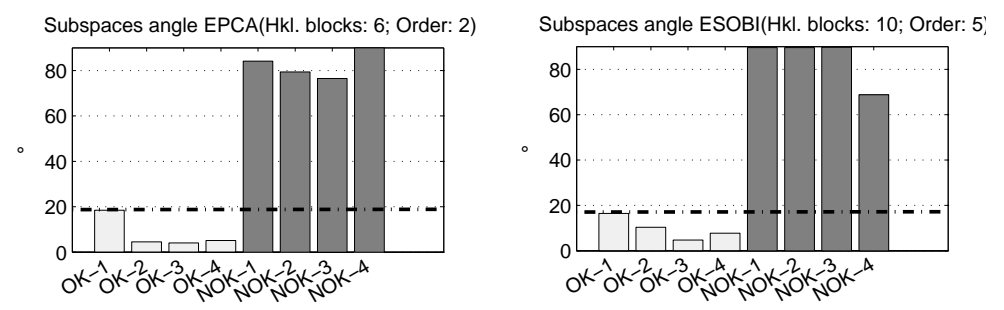

Fig. 1 Diagnosis of rotating devices with EPCA method on the right and ESOBI on the left.

Detection results are presented in figure 1 . The first healthy device (OK-0) is considered as the reference state. Both methods were able to make a clear distinction between the faulty devices and the healthy ones. 


\subsection{Quality control of weldings}

The second example involves an industrial welding machine from a steel processing plan. The machine was instrumented with a monoaxial accelerometer on the upper forging wheel. The purpose of this wheel is to flatten the welded joint during the welding process.

The quality of the welded joints depends on several parameters. In this example, four distinct parameters were altered and multiple alteration levels were considered, leading to a batch of 27 welded joints with out-of-range parameters. Six welded joints were also realized using nominal parameters for false-positive testing (Table 1). A microscopic quality control of each welded joint was realized at the end of the campaign to assess their actual quality.

Table 1. Welding parameters during the measurement campaign

\begin{tabular}{|c|c|c|c|}
\hline Welding Name & Parameter & $\mathrm{Nbr}$. of samples & Weld quality \\
\hline OKK & Nominanal levevel & 6 & Good . . . . \\
\hline A & $-33 \%$ covering & 3 & Acceptable \\
\hline B & . $66 \%$ covering & 3 & .... Bad . . . \\
\hline $\mathrm{C}$ & . $33 \%$ compensation & 3 & Good \\
\hline D & -66\% compensation & 3 & Acceptable \\
\hline E & $-10 \%$ current & 3 & Acceptable \\
\hline F & $-20 \%$ current & 3 & $\ldots$. . . Bad . . . \\
\hline G & $-10 \%$ forging pressure & 3 & . . . Good _. . \\
\hline H & $+5 \%$ forging pressure & 3 & Acceptable \\
\hline $\mathrm{I}$ & $-66 \%$ covering and compensation & 3 & $\mathrm{Bad}$ \\
\hline
\end{tabular}

The detection results of EPCA and ESOBI are presented in figure 2.
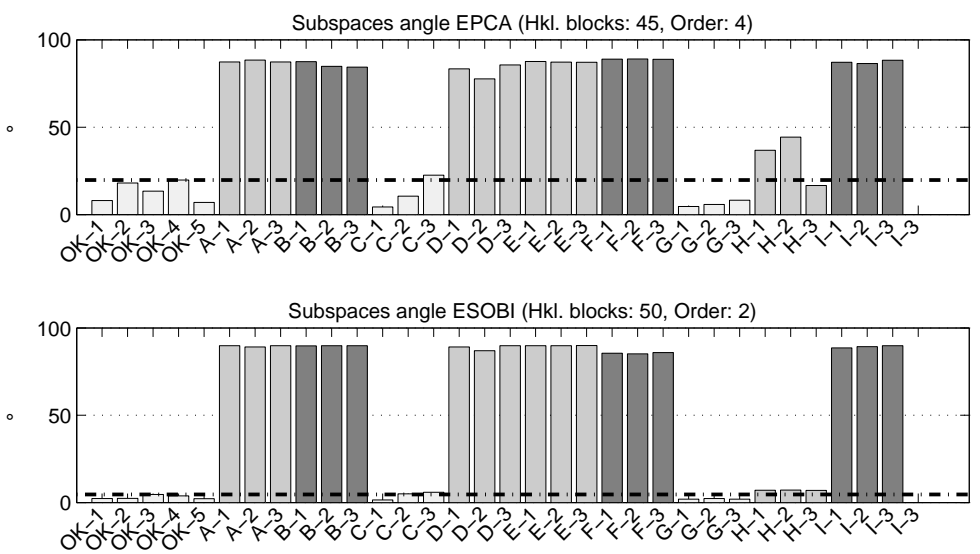

Fig. 2. Faulty welded joint detection results by EPCA (top) and ESOBI (bottom) 
The detection results are good. The subspace angles by ESOBI seem to be more consistent than EPCA.

\section{Conclusion}

The presented methods where successfully applied to damage detection problems in industrial environment. By making use of the Hankel matrices, only one sensor is needed for the diagnosis which is an appreciable advantage.

Acknowledgments This work is supported by the "Mint Project" funded by the "Region Wallone" of Belgium which is greatly acknowledged.

\section{References}

1. Poncelet F., Kerschen G., Golinval J.C., Verhelst D.: Output-only modal analysis using blind source separation techniques. Mechanical Systems and Signal Processing, 21, 2007, pp. 2335-2358

2. De Boe P., Golinval J.C.: Principal component analysis of a piezosensor array for damage localization, Structural Health Monitoring, 2003, pp. 137-144

3. Belouchrani A., Abed-Meraim K., Cardoso J.F., Molines E.: A blind source separation technique using second order statistics, IEEE transactions on signal processing, 45, 1997, pp. 434-444

4. Nguyen V.H.: Damage Detection and Fault Diagnosis in Mechanical Systems using Vibration Signals, PhD Thesis, 2010,

5. Van Overschee P., De Moor B.: Subspace identification for linear systems-TheoryImplementation-Applications, Kluwer Academic Publishers, 1997

6. Golub G.H., Van Loan C.F.: Matrix computations ( $3^{\text {rd }}$ edition), The John Hopkins University press, 1996

7. Yan A.M., Golinval J.C.: Null subspace based detection of structures using vibration measurements, Mechanical Systems and Signal Processing, 20, 2006, pp. 611-626

8. Rutten C., Loffet C., Golinval J.C.: Damage detection of mechanical components using null subspace analysis, European Journal of Mechanical and Environmental Engineering, 2009 